University of Wollongong

Research Online

Australian Institute for Innovative Materials -

Papers

Australian Institute for Innovative Materials

$1-1-2013$

\title{
In vitro growth and differentiation of primary myoblasts on thiophene based conducting polymers
}

Anita F. Quigley

University of Wollongong, anitaq@uow.edu.au

Klaudia K. Wagner

University of Wollongong, kwagner@uow.edu.au

Magdalena Kita

University of Wollongong, mkita@uow.edu.au

Kerry J. Gilmore

University of Wollongong, kerryg@uow.edu.au

Michael J. Higgins

University of Wollongong, mhiggins@uow.edu.au

See next page for additional authors

Follow this and additional works at: https://ro.uow.edu.au/aiimpapers

Part of the Engineering Commons, and the Physical Sciences and Mathematics Commons

Research Online is the open access institutional repository for the University of Wollongong. For further information contact the UOW Library: research-pubs@uow.edu.au 


\title{
In vitro growth and differentiation of primary myoblasts on thiophene based conducting polymers
}

\begin{abstract}
Polythiophenes are attractive candidate polymers for use in synthetic cell scaffolds as they are amenable to modification of functional groups as a means by which to increase biocompatibility. In the current study we analysed the physical properties and response of primary myoblasts to three thiophene polymers synthesized from either a basic bithiophene monomer or from one of two different thiophene monomers with alkoxy functional groups. In addition, the effect of the dopants pTS- and $\mathrm{ClO} 4$ - was investigated. In general, it was found that pTS- doped polymers were significantly smoother and tended to be more hydrophilic than their $\mathrm{ClO} 4$ - doped counterparts, demonstrating that the choice of dopant significantly affects the polythiophene physical properties. These properties had a significant effect on the response of primary myoblasts to the polymer surfaces; LDH activity measured from cells harvested at 24 and $48 \mathrm{~h}$ post-seeding revealed significant differences between numbers of cells attaching to the different thiophene polymers, whilst all of the polymers equally supported cell doubling over the $48 \mathrm{~h}$ period. Differences in morphology were also observed, with reduced cell spreading observed on polymers with alkoxy groups. In addition, significant differences were seen in the polymers' ability to support myoblast fusion. In general pTS- doped polymers were better able to support fusion than their $\mathrm{ClO} 4$ doped counterparts. These studies demonstrate that modification of thiophene polymers can be used to promote specific cellular response (e.g. proliferation over differentiation) without the use of biological agents. 2013 The Royal Society of Chemistry.
\end{abstract}

\section{Keywords}

growth, polymers, vitro, conducting, differentiation, thiophene, myoblasts, primary

Disciplines

Engineering | Physical Sciences and Mathematics

\section{Publication Details}

Quigley, A. F., Wagner, K. K., Kita, M., Gilmore, K. J., Higgins, M. J., Breukers, R. D., Moulton, S. E., Clark, G. M., Penington, A., Wallace, G. G., Officer, D. L. \& Kapsa, R. M. (2013). In vitro growth and differentiation of primary myoblasts on thiophene based conducting polymers. Biomaterials Science, 1 (9), 983-995.

\section{Authors}

Anita F. Quigley, Klaudia K. Wagner, Magdalena Kita, Kerry J. Gilmore, Michael J. Higgins, Robert D. Breukers, Simon E. Moulton, Graeme M. Clark, Anthony Penington, Gordon G. Wallace, David L. Officer, and Robert M. Kapsa 
In vitro growth and differentiation of murine primary myoblasts on thiophene based conducting polymers.

Anita F. Quigley ${ }^{\mathrm{a}, \mathrm{b}}$, Klaudia Wagner ${ }^{\mathrm{a}}$, Magdalena Kita ${ }^{\mathrm{a}, \mathrm{b}}$, Kerry J. Gilmore ${ }^{\mathrm{a}}$, Michael J. Higgins ${ }^{\mathrm{a}}$,

Robert D. Breukers ${ }^{\mathrm{a}}$, Simon E. Moulton ${ }^{\mathrm{a}}$, Graeme M. Clark ${ }^{\mathrm{a}, 1}$, Anthony J. Penington ${ }^{\mathrm{c}}$, Gordon G. Wallace $^{\mathrm{a}}$, David L. Officer ${ }^{\mathrm{a}}$, Robert M. I. Kapsa ${ }^{\mathrm{a}, \mathrm{b}, \mathrm{c}, *}$.

${ }^{a}$ ARC Centre of Excellence for Electromaterials Science, Intelligent Polymer Research Institute, University of Wollongong, Northfields Avenue, Wollongong, NSW 2522, Australia.

${ }^{\mathrm{b}}$ Centre for Clinical Neuroscience and Neurology Research, St. Vincent’s Hospital, 41 Victoria Pde., Fitzroy, VIC 3065, Australia.

'Department of Medicine, University of Melbourne, St. Vincent’s Hospital, 41 Victoria Pde, Fitzroy, VIC 3065, Australia

*Corresponding Author: A/Prof Robert M. I. Kapsa

Centre for Clinical Neuroscience and Neurology Research,

$5^{\mathrm{TH}}$ Floor Daly Wing,

St. Vincent's Hospital,

41 Victoria Pde.,

Fitzroy, VIC 3065, Australia.

rmik@unimelb.edu.au

Ph: +61 392883344

Fax: +61 392883350

\footnotetext{
${ }^{1}$ Present address: NICTA, Department of Electrical and Electronic Engineering, University of Melbourne, Victoria, 3010, Australia.
} 


\begin{abstract}
Polythiophenes are attractive candidate polymers for use in synthetic cell scaffolds as they are amenable to modification of functional groups as a means by which to increase biocompatibility. In the current study we analysed the physical properties and response of primary myoblasts to three thiophene polymers synthesized from either a basic bithiophene monomer or from one of two different thiophene monomers with alkoxy functional groups. In addition, the effect of the dopants $p \mathrm{TS}^{-}$and $\mathrm{ClO}_{4}^{-}$was investigated. In general, it was found that $p \mathrm{TS}^{-}$doped polymers were significantly smoother and tended to be more hydrophilic than their $\mathrm{ClO}_{4}{ }^{-}$doped counterparts, demonstrating that the choice of dopant significantly affects the polythiophene physical properties.

These properties had a significant effect on the response of primary myoblasts to the polymer surfaces; significant differences were seen in the rate of cell division, as determined by LDH activity. Differences in morphology were also observed, with reduced cell spreading observed on polymers with alkoxy groups. In addition, significant differences were seen in the polymers' ability to support myoblast fusion. In general $\mathrm{pTS}^{-}$doped polymers were better able to support fusion than their $\mathrm{ClO}_{4}{ }^{-}$doped counterparts. These studies demonstrate that thiophene polymers can be tailored to obtain the desired cellular response (e.g. proliferation over differentiation) without the use of biological agents.
\end{abstract}

Keywords: Skeletal Muscle, Myoblast, Polythiophene, Differentiation, Roughness 


\section{Introduction}

Effective engineering of muscle tissue facilitates the remodeling of tissues lost due to disease, trauma or surgery and as such, can involve replacement of significant amounts of tissue. To date, muscle remodeling approaches involving cell replacement have shown limited potential due to a variety of issues, including death and/or immune rejection of donor cells, lack of donor cell proliferation and limited migration from the injection site [1;2]. Thus many cell transplantation strategies designed to treat severe loss of muscle tissue in conditions such as Duchenne Muscular Dystrophy or trauma, have achieved limited degrees of success [3;4].

A number of reports have highlighted the diversity of approaches that have been applied to improve skeletal muscle engineering, including the use of micro-patterned polymers [5-7], biological scaffolds [8] and synthetic polymers [9-13]. Synthetic scaffolds have the advantage over biologic scaffolds in that their characteristics can be defined and manipulated to be inherently pro-myogenic (i.e. “myo-bionic”), thus greatly facilitating their utility in future bionic approaches for skeletal muscle engineering. Such engineered scaffolds may be seeded ex vivo with host or donor cells [7] and implanted, or utilized directly in vivo to encourage endogenous muscle regeneration by influencing myoblast division and migration, without the need for cell replacement or cell seeding.

Organic conducting polymers (OCPs) such as polypyrrole, polyaniline and pEDOT have demonstrated reasonable biocompatibility $[14 ; 15]$ which has been shown to vary according to the counter-ion used as the dopant, the polymerisation conditions and resulting properties (i.e. surface roughness, surface energy etc.) [16]. OCPs have been shown to support myoblast adhesion and proliferation [17;18], and their facilitation of electrical stimulation [19] and controlled release of functional proteins to influence cell growth in vitro and in vivo [20;21] offers an exciting new approach for influencing skeletal muscle regeneration.

As an “excitable” tissue, muscle and its resident progenitor cells, myoblasts, have been shown to respond to electrical stimuli both in vitro and in vivo, influencing proliferation [22] as well as differentiation [23;24]. OCPs thus have potential applications in effecting control of myoblast 
proliferation and/or differentiation directly as part of implantable scaffold/electrode systems. Alternatively, OCPs may provide a substratum as part of a "myo-reactor” system for ex vivo preconditioning of myogenic precursors to optimal regenerative condition prior to implantation [7].

Polythiophenes are attractive candidate polymers for use in synthetic cell scaffolds as they are more amenable to modification of functional groups as a means by which to increase biocompatibility than are other OCPs such as polyaniline and polypyrrole. This is highlighted by the synthetic methods that have been described for the introduction of a wide variety of functional groups to polythiophenes [25]. Despite this, there are few reports describing the modification and use of polythophenes for cellular interaction other than for pEDOT [5;18;26-28].

Our group has recently investigated the use of an ester-functionalized polythiophene and its hydrolyzed derivative, demonstrating excellent capacity to support myoblast proliferation and differentiation in vitro [12]. In addition, this organic solvent-soluble polythiophene was electrospun to provide an aligned nanostructured scaffold that orientated myoblast differentiation, in vitro. We expand on these findings in the current study, where we investigate the capacity of three different polythiophenes to support primary myoblast proliferation and differentiation and the effects of the polymer properties on myoblast behaviour. Poly(bithiophene) (PBTh) was chosen as a control polymer, to examine the effect of the basic polythiophene backbone. Two variants of the basic PBTh chemistry were synthesized by introduction of alkoxy substituents to i) improve the oxidative stability of the polymers and ii) provide molecular entities amenable to further functionalization with other molecules. An alkoxy-substituted polythiophene, poly-3-decoxythiophene (P3DTh) was synthesized to explore the effect of the solubilizing functionality that would be required for a processable polymer. The third polymer, poly(decyl 4,4'’-didecoxy-2,2':5',2' '-terthiophene-3'carboxylate, PDTTh3E), incorporates both the solubilizing groups as well as an ester group amenable to subsequent functionalization with peptides. We explored the physical properties of the resulting polythiophene films synthesized with two different dopants, tetrabutyl ammonium paratoluene sulphonate (pTS $\left.{ }^{-}\right)$and tetra butyl ammonium perchlorate $\left(\mathrm{ClO}_{4}^{-}\right)$to provide surface energy variants in the first instance and conductivity variants to allow subsequent electrical 
stimulation studies. In this study, these polymers were evaluated for their influence on the growth and differentiation of primary myoblasts.

\section{Methods}

2.1 Polythiophene film synthesis, electrochemistry and spectroscopic characterization.

The monomers 3-decoxy thiophene (3DTh) and decyl 4,4’’-didecoxy-2,2':5’,2’terthiophene-3'-carboxylate (terthiophene) (DTTh3E) were synthesized essentially as described by Gambhir and Feldhues [25;29]. The polymers films were synthesized galvanostatically at a current density of $1 \mathrm{~mA} / \mathrm{cm}^{2}$ on Au-Mylar (CP Films Inc.) in two different electrolytes: $0.1 \mathrm{M}$ tetrabutyl ammonium perchlorate $\left(\mathrm{ClO}_{4}^{-}\right)$or tetrabutyl ammonium paratoluene sulphonate (pTS) (Aldrich). The Au-Mylar was cleaned, prior to deposition, by sonication for 2 min in the following solutions: detergent (Triton X-100; 0.05\%) water, isopropanol and methanol. Electrochemical polymerization of bithiophene (Aldrich) and 3DTh was carried out from solutions containing $0.1 \mathrm{M}$ of the monomer in acetonitrile for 40s and 20s respectively. In the case of DTTh3E the concentration of the monomer was $20 \mathrm{mM}$ in a 1:1 mixture of dichloromethane and acetonitrile and polymerization was carried out for 20s. After synthesis all films were rinsed in acetonitrile. All electrochemical experiments were performed in a three-electrode cell, with a non-aqueous $\mathrm{Ag} / \mathrm{Ag}^{+}$reference and Pt mesh counter electrode, using an eDAQ system controlled by EChem software. Absorbance spectra were recorded using a Shimadzu UV-1800 spectrophotometer.

\subsection{Water contact angle}

The wettability of the films was characterized using a Data Physics OCA20 goniometer. Contact angle was measured using the sessile drop method ( $2 \mu \mathrm{L}$, Milli Q water). Contact angles were determined under a number of conditions including directly after deposition, rinsing and drying; after 24 hours exposure to air or after 19 hrs. incubation in cell culture media at room 
temperature or $24 \mathrm{~h}$ incubation at $37^{\circ} \mathrm{C}$. In each case an average of 5 measurements \pm standard deviation was calculated.

\subsection{Atomic Force Microscopy (AFM)}

Polymer samples were prepared for characterisation by washing with Milli Q water and drying with nitrogen gas. AFM imaging was performed using a JPK Biowizard II AFM (JPK Instruments, Germany). AFM images were obtained in air at $25^{\circ} \mathrm{C}$ using a $0.29 \mathrm{~N} / \mathrm{m}$ silicon nitride cantilever in tapping mode with a scan rate of 0.5 Hz. Root Mean Square (R.M.S) roughness values were calculated from AFM height images using analysis software of the AFM.

\subsection{Myoblast Cell Culture}

Bl10.129S4Gt(ROSA)26 mice (Bioresources Center, St. Vincent’s Hospital, Melbourne, Australia) were exposed to a 12 hour day/night cycle and fed ad libitum until they were sacrificed for myoblast culture at 5-6 weeks of age. All animal handling was performed according to St Vincent’s Hospital Animal Ethics Committee protocol 86/06 in accordance with the Australian Code of Practice for the Care of Animals for Scientific Purposes (NHMRC).

After cervical dislocation, skeletal muscle was removed from the hind limbs of mice and primary myoblast cultures prepared essentially as previously described [30]. Adherent myoblasts were cultured in proliferation medium (Hams/F10 (Trace Biosciences) supplemented with 20\% fetal

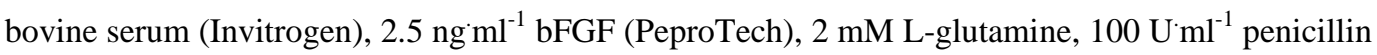
and $100 \mu \mathrm{gml}^{-1}$ streptomycin (Invitrogen)). All cell culture was carried out in $5 \% \mathrm{CO}_{2}$ at $37^{\circ} \mathrm{C}$.

\subsection{Quantification of Cell Adhesion}

Circular discs of polythiophene films and Au mylar (6 mm in diameter) were placed into polystyrene 96-well plates (Nunc). Polymer surfaces were sterilized by briefly washing with 70\% ethanol and air drying before removal of residual ethanol by soaking in 2 washes of serum-free culture media. Myoblasts were then seeded in proliferation media (100 $\mu \mathrm{l} /$ well $)$ at a density of Page 6 
15,000 cells $/ \mathrm{cm}^{-2}$. Cells were allowed to adhere for 2,24 and 48 hrs. to determine initial attachment (2 h) and rate of proliferation (24 and 48 h). Before quantification of attached cells by Lactate Dehydrogenase (LDH) assay, the media containing non-adherent cells was removed and the films washed briefly with proliferation media. The remaining attached cells were then assayed for LDH activity using a CytoTox96 Non-Radioactive Cytotoxicity Assay (Promega), according to manufacturer’s instructions. Results were analyzed for significance by one-way ANOVA with Bonferroni post-hoc analysis (GraphPad Prism V 5.0.4).

\subsection{Differentiation of myoblasts to myotubes}

As described above, circular discs of polythiophene films and Au mylar (5 mm diameter) were placed into sterile polystyrene 96-well plates (Nunc) and sterilized. Myoblasts were seeded in proliferation media at 30,000 cells/ $\mathrm{cm}^{2}$ and allowed to adhere for $24 \mathrm{~h}$. Myotube differentiation was then induced by changing the media to DMEM supplemented with $2 \%(\mathrm{v} / \mathrm{v})$ horse serum, $4 \mathrm{mM} \mathrm{L-}$ glutamine, 100 units $\mathrm{ml}^{-1}$ of penicillin and streptomycin (all reagents from Invitrogen). Myoblasts were allowed to differentiate for 3 days before further analysis was made.

\subsection{Analysis of myoblasts and myotube differentiation.}

Myoblasts were seeded onto polythiophene disks, as outlined above, and allowed to proliferate under standard tissue culture conditions for 48 hrs. for visualization of actin filaments and Proliferating Cell Nuclear Antigen (PCNA) expression. For phalloidin labelling of actin filaments, the media was removed and cells fixed in 4\% paraformaldehyde (Sigma) for 15 minutes before labelling with AlexaFluor 488 Phalloidin (Invitrogen), according to manufacturer's specifications. Cell nuclei were then stained with $1 \mathrm{ug}^{\prime} \mathrm{ml}^{-1}$ DAPI (4,6-diamidino-2-phenylindole dihydrochloride, Sigma) for 5 mins and specimens mounted in fluorescent mounting media (Dako) for microscopy.

For PCNA immunostaining, the cells were fixed with 100\% methanol (BDH) for 15 mins, washed in phosphate-buffered saline (PBS, pH 7.4) and permeabilized with $0.01 \%$ Triton-x 100 (Sigma). Non-specific sites were blocked in 10\% donkey serum (Millipore) in PBS (Sigma) for 60 
mins, then incubated with mouse anti-PCNA (Sigma) at 1:1000 dilution in blocking solution for 60 mins at $37^{\circ} \mathrm{C}$, followed by two washes with PBS. Cells were then incubated with donkey antimouse Alexa Fluor 594 (Invitrogen) diluted 1:2000 in blocking solution for 60 minutes at $37^{\circ} \mathrm{C}$. Cell nuclei were stained with DAPI and mounted for microscopy as described above.

Differentiating myoblasts were stained for desmin expression as follows. After fixation with 100\% ice cold methanol (BDH) for 15 mins and washing in PBS, cells were immunolabelled as described above using a mouse anti-desmin monoclonal antibody (NovoCastra) at 1:100 dilution followed by donkey anti-mouse Alexa Fluor 594 (Invitrogen). Nuclei were labelled with DAPI and processed for microscopy as described above. All specimens were visualized using an Olympus IX70 fluorescent microscope and images obtained using Spot Advanced 4.0.9 software (Diagnostic Instruments). Random non-overlapping fields of fluorescently labelled myotubes were taken at 100x magnification. Myotube differentiation was assessed by calculating the percentage of nuclei within myotubes (fusion of two or more myoblasts) as a percentage of total nuclei (from both myoblasts and myotubes) per field. Cell numbers were quantified using the cell count function in ImageJ (NIH).[31]

\subsection{Statistical analyses}

Analysis of LDH activity and cell differentiation were carried out using one way ANOVA with Bonferroni post-hoc analysis. Linear regression analysis was used to determine the relationship between nuclei $/ \mathrm{cm}^{2}$ and degree of multinucleation. Non-linear relationships (i.e. correlations with RMS and Contact Angle) were analyzed using parametric two-tailed Pearsons Product-Moment Correlation analysis. All analyses were carried out using GraphPad Prism (Version 6.0.1)

\section{Results}

\subsection{Polythiophene film synthesis}

Bithiophene (PBTh), alkoxy-derived thiophene (P3DTh) and an ester functionalized terthiophene (PDTTh3E) were polymerised by galvanostatic deposition with either $\mathrm{ClO}_{4}{ }^{-}$or $p \mathrm{TS}^{-}$as dopants. In 
all cases, coherent uniform films were deposited at constant current density. Deposition times were short in order to limit the polymer film thickness to give sufficient transparency for optical microscopy.

\subsection{Surface hydrophobicity}

Water contact angle measurements of all polythiophene films deposited on Au-Mylar and ITO were measured under the following conditions: just after electrodeposition, after $24 \mathrm{~h}$ in air (for films on ITO), after $19 \mathrm{~h}$ soaking in DMEM (CM1) and after $24 \mathrm{~h}$ soaked at $37^{\circ} \mathrm{C}$ in HAMS F10 media (CM2). The results are summarized in Table 1. The hydrophobicity of the films electrodeposited on Au-Mylar was measured using goniometry, with supplementary results from films deposited on ITO providing further insights into the films' de-doping dynamics by UV-visible spectroscopy.

The use of two different dopants for each polymer resulted in significantly different contact angles. $\mathrm{ClO}_{4}^{-}$polymers initially tended to be more hydrophobic than $p \mathrm{TS}^{-}$polymers, when the polymer has hydrophobic substituents. For unsubstituted PBTh, this trend was reversed. The variation in contact angle of both P3DTh and PDTTh3E was much smaller between dopants than that seen for PBTh, for which both $\mathrm{CLO}_{4^{-}}$and pTS variants remained hydrophilic. It must be noted however, that the films were fully oxidized in solution before the first measurement. As discussed in the next section, spontaneous reduction (de-doping) of the films over the course of the contact angle measurements reduced the influence of the dopant.

\subsection{Polymer film oxidative stability}

To probe the oxidative stability of the polymer films in the dry state and after exposure to culture media, the PBTh, P3DTh and PDTTh3E polymer films were examined by UV-vis spectroscopy. Figure 1 shows representative UV-vis spectra, since all the polymer films showed similar characteristics. This series of spectra from $\mathrm{PBTh}_{-} \mathrm{ClO}_{4}$, recorded just after electrodeposition and then after exposure of the film to air and culture media, are generally representative of the other 
thiophene/dopant species evaluated in this study. Very broad bands between 600 to $1500 \mathrm{~nm}$, characteristic of polaron (or bipolaron) absorption, can be seen for the freshly prepared film (Fig. 1, black line). After exposure to air for $24 \mathrm{~h}$, these bands reduced in intensity, while the peak at $490 \mathrm{~nm}$ corresponding to the neutral polythiophene increased. Interactions of the films with culture media were shown to accelerate these changes, indicative of polymer reduction. The corresponding increase in PBTh film hydrophobicity with dopant loss is evident in the contact angle measurements (Table 1).

\subsection{Atomic Force Microscopy (AFM): Surface Topography}

Initial attempts were made to obtain AFM images of the polymer surfaces in solution so as to more accurately reflect the actual surfaces exposed to cells within a cell culture environment. However, the nodular and friable nature of the surfaces, particularly the PBTh, prevented wet environmental surface measurement and consequently, only dry surface measurements were made. The surface roughness of the films, measured within 100 and $10 \mu \mathrm{m}$ squares, on each of the respective films is shown in Table 1. Images made within $10 \mu \mathrm{m}$ squares are presented in Figures 2A-F. Polymers synthesized with $\mathrm{ClO}_{4}{ }^{-}$as the counter ion had a general morphology that consisted of a continuous underlying film (i.e. primary surface features) with particulate structures (secondary features) on the surface. The roughness of each film was primarily determined by the number and size of these particulate surface structures. The particulate structures of the PBTh were not well adhered to the smooth underlying film and were easily swept away by the AFM tip during imaging (results not shown). At $100 \mu \mathrm{m}$ resolution, $\mathrm{PBTh}_{-\mathrm{ClO}}$ and $\mathrm{PDTTh}_{3} \mathrm{E}-\mathrm{ClO}_{4}$ were both found to be relatively rough with RMS of 574.2 and $585.9 \mathrm{~nm}$ respectively. $\mathrm{P} 3 \mathrm{DTh}-\mathrm{ClO}_{4}$ was relatively smoother with a RMS of $217.0 \mathrm{~nm}$ at $100 \mu \mathrm{m}$ resolution (Fig. 2C).

The use of $\mathrm{TTS}^{-}$as a dopant resulted in the production of smoother film surfaces (Figs. 2B, D and F; Table 1), when compared to $\mathrm{ClO}_{4}{ }^{-}$doped polymers, which could also influence their wettability. This effect reached statistical significance $(p<0.05)$ over $100 \mu \mathrm{m}$ areas (Fig. $2 \mathrm{G})$. A similar trend (not statistically significant) was evident at $10 \mu \mathrm{m}$ resolution. In the $p$ TS doped group, 
the smoothest polymer was PBTh-pTS, followed by P3DTh-pTS and then PDTTh3E,-pTS at both 10 and $100 \mu \mathrm{m}$ (see Table 1).

\subsection{Adhesion and proliferative metabolism of myoblasts}

The affinity of myoblasts for the polythiophene films was assessed by quantification of Lactate Dehydrogenase (LDH) activity measured in attached cells at 2, 24 and 48 hrs.. LDH was used as an indicator of relative cell numbers on the basis of its constitutive expression as a metabolic enzyme component of the glycolytic cycle. The results for initial cell adhesion, analyzed at $2 \mathrm{~h}$ post cell seeding, show that myoblasts adhere to the range of polymer films included in this study to a similar extent, with no significant difference between cellular LDH activities detected on the respective substrates (including tissue culture polystyrene (PS) or Au-mylar control surfaces, data not shown). At 24 hrs. post seeding, lower LDH activities were evident in cell populations grown on PBTh- $\mathrm{ClO}_{4}$ and PDTTh3E-pTS ( $\left.<<0.05\right)$ compared to the other polymer formulations (Fig. 3A), indicative of either reduced cell numbers or otherwise reduced cellular metabolic activity.

By 48 hrs., differences between the cells respective affinities for the polymers became more apparent. P3DTh-pTS yielded cells with the highest mean LDH activity overall (indicating the largest numbers of cells attached), significantly higher than on PBTh- $p \mathrm{TS}(p<0.01)$, or PBTh-ClO $(\mathrm{p}<0.001)$ (Fig. 3B). Cells grown on $\mathrm{PBTh}_{-} \mathrm{ClO}_{4}$ yielded significantly lower LDH activity than on all other polymers tested with the exception of PBTh-pTS. In the $\mathrm{ClO}_{4}^{-}$doped group, $\mathrm{P} \mathrm{DTh}-\mathrm{ClO}_{4}$ and PDTTh3E-ClO ${ }_{4}$ demonstrated better cell attachment performing equally well at $48 \mathrm{~h}$, both with significantly higher LDH activity than $\mathrm{PBTh}^{-\mathrm{ClO}_{4}}(p<0.001)$. The $\mathrm{LDH}$ activity recovered from myoblasts on all of the respective polymer surfaces was doubled at $48 \mathrm{~h}$ from that at $24 \mathrm{~h}$, indicating that all of the polymers equally facilitated metabolic expansion of attached myoblasts. In agreement with this, PCNA staining at 48 hrs. revealed that at least a sub-population of myoblasts on each surface were actively proliferating (Supplementary Fig. 1).

Actin labeling with phalloidin-Alexafluor 488 demonstrated some variation in cell morphology. The majority of cells on PBTh showed a stellate morphology (Figs. 4A and B) similar 
to that observed on the control Au film, while more rounded cells were apparent on P3DTh and PDTTh3E (Fig. 4C-F). However, whilst this may be an indicator of generally more favorable proliferative status on the P3DTh and PDTTh3E species, specific detailed quantitative confirmation of this aspect of myogenic cell growth behavior fell beyond the scope of this study and will be reported elsewhere.

\subsection{Differentiation of myoblasts to myotubes}

Myoblasts were cultured in growth medium on polythiophene films and control (PS and Au) substrata for $24 \mathrm{~h}$ and then induced to differentiate into myotubes for $72 \mathrm{~h}$ in low serum medium. The cells were fixed and immunostained and myoblast differentiation quantified by scoring the proportion of cell nuclei within multinucleate myotubes as a percentage of total nuclei per field.

Desmin immunolabelling and DAPI nuclear staining demonstrated that myotubes and undifferentiated myoblasts were present on all the polymer films (Fig. 5A-G), however there was significant variation in myotube formation between polymer types. Tissue culture polystyrene (PS) and Au-Mylar were used as reference substrata for myoblast differentiation, having shown satisfactory facilitation of myoblasts in previous experiments. No significant difference in myoblast differentiation was observed on these two substrata, with both supporting multinucleation (myotube formation) at approximately 64\% (Fig. 5H). Only two of the tested polymers, PTTh3E-ClO $(p<0.001)$ and $\mathrm{P} \mathrm{DTh}-\mathrm{ClO}_{4}(p<0.05)$ showed significantly lower percentage myoblast differentiation than these controls. Further to this, myoblasts differentiated on $\mathrm{ClO}_{4}{ }^{-}$doped polymers generally showed lower levels of multinucleation compared to myoblasts differentiated on their $p \mathrm{TS}^{-}$doped counterparts (Fig. 5H), however this trend only reached statistical significance on PPTTh3E films $(p<0.01)$ films. In the $\mathrm{ClO}_{4}{ }^{-}$group of polymers, $\mathrm{PBTh}_{-} \mathrm{ClO}_{4}$ showed the highest level of myotube formation, reaching significance when compared to PTTh3E-ClO $4(p<0.001)$, which showed significantly lower capacity to support myotube formation compared to all the other substrata included in this study. The same trend was observed for $p \mathrm{TS}^{-}$doped polymers, however the reduced 
myotube formation on PPTTh3E-pTS films was not statistically different to that on the remaining pTS $^{-}$polymers.

The order of progression from highest to lowest degree of myotube formation on pTh doped with either of the two counter-ions was PBTh>P3DTh>PDTTh3E (Fig. 5H). Myoblasts grown on PBTh underwent the highest levels of myoblast differentiation overall. However, this polymer chemistry supported the lowest numbers of myoblasts by 48 h (Fig. 3), further supporting a biomimetic role for the PBTh polymer chemistry towards induction of myogenic differentiation compared to the other thiophene formulations used here.

Nuclear density was analysed by counting the total nuclei present in myoblasts that were subjected to $24 \mathrm{~h}$ of attachment in growth media, followed by 3 days of differentiation on the polymers in low serum media. The densities of DAPI-stained nuclei (nuclei/ $\mathrm{cm}^{2}$ ) were calculated for each polymer (Fig. 6A). Not unexpectedly, this revealed a similar profile to that observed for myoblast differentiation (Fig. $5 \mathrm{H})$, yielding a significant correlation $(R=0.87, p<0.03$; $P$ ) between cell density (nuclei/cm²) and myotube formation (Fig. 6B). In all cases, $p \mathrm{TS}^{-}$doped polymers facilitated significantly higher cell densities compared to their $\mathrm{ClO}_{4}{ }^{-}$doped counterparts, under differentiation conditions. In both dopant series, the PBTh substrata yielded higher nuclear densities under differentiation conditions (low serum) than did the other two thiophene species used. This suggests that at least in part, the higher myo-differentiation propensity of myoblasts grown on PBTh species suggested by other data presented in this study (Figs 4 and 5H) may nevertheless involve some cell proliferation under differentiation conditions to critical contact densities that enhance differentiation. P3DTh-ClO 4 and $\mathrm{PDTTh} \mathrm{E}-\mathrm{ClO}_{4}$ supported significantly lower nuclei/ $\mathrm{cm}^{2}$ under differentiation conditions than control tissue culture PS (Fig 6), used as a control substrate ( $p<0.05$ and $p<0.001$ respectively) whilst PBTh-pTS supported the highest nuclear density of all of the formulations, with the exception of the P3DTh-pTS substratum.

\subsection{Effect of Polymer Properties on Myoblast and Myotube Behaviour}


In order to gain some understanding of the effects of polymer properties on cell behavior, contact angle and roughness (RMS) were correlated with myoblast LDH activity, multinucleation and nuclear density after differentiation. Contact angle did not show a significant correlation with roughness although a trend was observed at both $10 \mathrm{um}$ and $100 \mathrm{um}$ resolution (data not shown). Likewise, myoblast LDH activity did not show a significant correlation with roughness (data not shown), however significant correlations were observed between LDH activity after $48 \mathrm{~h}$ and both contact angle measured on freshly prepared polymers $\left(\mathrm{t}=0 ; R^{2}=0.73, p<0.03\right)$ and polymers exposed to DMEM for $19 \mathrm{~h}\left(\mathrm{t}=19 \mathrm{~h} ; R^{2}=0.73, p<0.03\right.$, Fig. $\left.7 \mathrm{a}\right)$. These data both yielded peak myoblast adhesion on surfaces with contact angles approaching $\sim 90^{\circ}$. Furthermore, the general similarity of the relationship displayed on the thiophene surfaces immediately after deposition and after $19 \mathrm{~h}$ in DMEM indicates that evaluation of this relationship is equally valid on dry and biological mediawetted surfaces respectively.

Myoblast differentiation (represented by \% multinucleation) was not found to correlate significantly with surface contact angle, although a trend approaching significance $(\mathrm{p}<0.059$; Fig. $7 \mathrm{~b})$ was evident. Again, a striking similarity between the relational plot dynamics displayed by cells differentiating on thiophene surfaces in dry and wet (after 19 h immersion in DMEM) surfaces suggests that this evaluation is again equally effective on dry as on wet polymers.

A semi-logarithmic trend also approaching statistical significance $(\mathrm{p}<0.059$; Fig 7C) was observed between surface roughness (RMS) measured within a $100 \mu \mathrm{m}$ square and percentage of cells that underwent multinucleation on the thiophene surfaces. This trend progressed to a statistically significant correlation ( $\mathrm{p}<0.02)$ between RMS and post-differentiation nuclear density (Fig. 7D). Collectively, these data suggest that i) the polythiophene surfaces support a weak myonuclear differentiation effect over a range of surface roughnesses up to around 200 microns where this effect appears to plateau/alter (Fig 7C), and that ii) this effect correlates with an increased nuclear density during differentiation (7D). However, these interesting effects require more detailed investigation to fully develop the specific nature of surface topographical effects on myoblast attachment, proliferation and differentiation to myotubular form. 


\section{Discussion}

\subsection{Polymer properties.}

While a wide variety of synthetic polymers have been investigated for the engineering of muscle tissue, conducting polymers have distinct advantages in that they offer a platform for the simultaneous electrochemical, biological and mechanical stimulation of muscle cells.

Polythiophenes, in particular, can be readily synthetically modified to tailor important polymer properties such as morphology, surface energy and tensile strength[32]. Variation of substituents and dopants also enhances the versatility of these materials as tissue platforms.

In our previous investigations into the use of polythiophene substrates for the in vitro growth of skeletal muscle cells, we utilized two chemically prepared substituted polythiophenes of distinctly different surface characteristics[12]. It was demonstrated that, despite the diverse hydrophilic character of the films, both films supported the proliferation and differentiation of primary and transformed skeletal muscle myoblasts. In addition, we manipulated these polymers into aligned electrospun fibers for the orientation of differentiated primary myotubes, an important requirement for skeletal muscle engineering. However, this work raised a number of questions regarding the effect of the dopant on cell growth and differentiation, the stability of the conducting form of the polymer and the influence of the different substituents on cell performance.

In this investigation, we have attempted to probe these issues, using electrochemicallyprepared polymer films in which the dopant was varied, and alkoxy groups incorporated into the polymer backbone to enhance the oxidative stability of the polymer. The current study compares the properties of polymers synthesized from the electrochemical oxidation of three thiophene monomers, using both $\mathrm{ClO}_{4}{ }^{-}$and $p \mathrm{TS}^{-}$as counter anions and their effect(s) on the growth and differentiation of primary myoblasts in vitro. The different thiophene chemistries yielded a variety of surface energy (represented as from thesurface -contact angle of a water droplet) and roughness (surface RMS by AFM) effects between the different substituent groups. Likewise, variations in surface energy and roughness were also evident between the $\mathrm{ClO}_{4}{ }^{-}$and $p \mathrm{TS}^{-}$doped polymers (Table 1). 
Contact angle measurements were made on films exposed to air as well as cell culture media in order to investigate how the effects of media exposure might affect the surface available for cell growth (Table 1). With regard to the dopant, pTS $^{-}$doped thiophenes tended to be more hydrophilic than the analogous $\mathrm{ClO}_{4}{ }^{-}$doped polymers with the exception of PBTh, whose surfaces yielded the lowest contact angle measurements for both dopants. However, for both PBTh polymers, a reduction in hydrophilicity was observed over time either in air or culture media presumably due to de-doping. Spontaneous reduction with concomitant de-doping of the thiophene polymers under ambient conditions and in culture media has been reported previously [12;33]. In an attempt to improve the oxidative stability of the polymers, we introduced alkoxy substituents[34]. The UV-vis spectroscopic analysis of the films (Fig. 1) exposed to both air and culture media show, however, that the polythiophene films are still unstable in the oxidized form. This indicates partial de-doping, which can alter the surface wettability. Only in the case of the PBTh polymers (PBTh-ClO 4 and PBTh-pTS) was the increase in hydrophobicity clearly influenced by de-doping of the polymers, this has been observed previously by Kossmehl et al. [35]. The variation in contact angles for the other two polymers, P3DTh and PDTTh3E, for both dopants, is much smaller and does not follow any overt trend. The presence of the alkoxy groups thus appears to dominate the surface hydrophobicity, negating the effect of the dopant.

The morphology of the polymers was also clearly affected by the dopants with significant variations in surface roughness occurring between polymers (Fig. 2 and Table 1). All of the pTS doped polymers were much smoother than their $\mathrm{ClO}_{4}{ }^{-}$doped counterparts. In comparison with the chemically-prepared polythiophenes that we used in our previous study of muscle cell growth [12], these electrochemically grown films are up to five times rougher. Therefore, it was of interest to ascertain whether this increased roughness would have an impact on cell growth and differentiation as it is well documented that roughness, as well as wettability, can effect cell adhesion to and migration on substrata [36;37]

\subsection{Primary myoblast adhesion, proliferation and differentiation on thiophene polymers}


Primary myoblasts were seeded on the polymer surfaces for analysis of myoblast attachment, proliferation and differentiation. A general bio-affinity of the polymer surfaces for primary myoblasts was evident, with proliferative cellular metabolism occurring on all polymers for at least 48 hours. However, differences in cell number evident on the polymers by $48 \mathrm{~h}$ are nevertheless suggestive of some variation in proliferative metabolic rate imparted by polymer chemistry (Fig 3). Immunofluorescent staining of PCNA in the cells confirmed that proliferating cells were present on all polymers by $48 \mathrm{~h}$, indicating that progression though the cell cycle and effective cell division was taking place and in general all of the thiophene polymers evaluated in this study facilitated effective cell growth.

Despite this, extensive morphological differences were observed between cells proliferating on the different polymer types. On PBTh polymers, myoblasts generally demonstrated a stellate morphology, with well-defined actin filaments and showed similar morphology to cells grown on gold-coated mylar (Figs 4A, B and G). This morphology is characteristic of membrane lacunae formation and activation of stochastic radial filopodial extensions, suggested to indicate myoblast progression towards fusion and exit from proliferative cell cycling [38]. In contrast, significant numbers of the myoblasts grown on P3DTh and PDTTh3E polymers compared to the PBTh species appeared more rounded with contracted cytoplasm and generally condensed actin distribution, suggesting lower surface compatibility and reduced attachment and a more proliferative metabolic status. These observations are significant as actin is known to play an important role in myoblast adhesion, motility and fusion [39]. Extensive actin remodeling is required to promote effective myoblast alignment and fusion [40;41], and disruption of actin polymerization could potentially cause perturbation of the differentiation process.

This interpretation of morphological differences was indeed supported by higher myodifferentiation on PBTh surfaces (along with control Au-mylar and PS), compared to their-other likewise-doped polythiophene surfaces-counterparts used in this study-(Fig 5H) which demonstrated a lower capacity to support myoblast fusion and differentiation. However, the greater nuclear densities observed on the two PBTH dopant species compared to the other similarly doped thiophene 
species after differentiation in vitro (Fig 6A) suggest that the greater myo-differentiation on PBTh substrata compared to similarly doped P3DTh and PDTTh3E may involve a proliferative component (Fig 6B). Whilst the specific mechanisms behind this are unclear the results yielded in this study suggest that in addition to potential perturbation of actin polymerization, the greater prodifferentiation propensity of myoblasts grown on PBTh substrata may be mediated by PBTh chemistry's biomimetic support for proliferation and otherwise attainment of critical cell densities that enhance myo-differentiation response. In addition, obvious differences were evident between myogenic cell behaviors promoted by the polythiophene species doped with $\mathrm{ClO}_{4}{ }^{-}$and pTS respectively. In particular, the polythiophene species doped with pTS- supported greater differentiation (Fig 5H) and nuclear densities (Fig 6), than did the $\mathrm{ClO}_{4}^{-}$-doped polymers. This confirms polythiophene polymer chemistry as highly amenable to being tailored both by monomer derivatisation and by dopant chemistry to promote controllable effects on myoblast proliferation or differentiation.

\subsection{Effects of polymer properties on myoblast behavior}

Surface energy and roughness are important considerations in polymer chemistry, particularly for the interaction of biological agents and entities with polymer materials developed for bio-medical applications. The chemical and physical properties of surfaces are known to play a critical role in regulating cellular behavior. In particular surface energy and micro/nanoscale roughness have been shown to exert effects on cellular adhesion and proliferation, motility and differentiation [36;42;43]. These two properties are not mutually exclusive as the topographical features of a surface can have a large influence on the surface energy. A well-known example of this is the superhydrophobic lotus leaf effect which has been "mimicked" for synthesis of materials [44]. A general increase in contact angle with increasing roughness observed in this study, (with the exception of one polymer $\mathrm{PBTh}_{-} \mathrm{ClO}_{4}$ which exhibited high roughness but low contact angle) concurs with other reports (with some exceptions) of correlation of modulated roughness with hydrophobicity [45]. 
A significant correlation occurred between substratum contact angle and LDH activity recovered from cells on the surface suggesting an influence of polymer surface energy on myoblast adhesion (Fig 7A). The PBTh polymers presented a soft, powdery top layer that could be easily dislodged by the AFM stylus, a characteristic previously reported by our group [46;47]. This may have a bearing on the relatively low cell numbers observed on the PBTh polymers (both dopants) after $48 \mathrm{~h}$.

Myoblast adhesion and to a lesser extent differentiation were observed to be influenced by contact angle (CA), with optimal adhesion and differentiation occurring at CA of around $90^{\circ}$ (Fig 7A) and $60^{\circ}$ (Fig 7B) respectively. Surface roughness appeared to show an inverse relationship trend with differentiation (Fig 7C), along with an inverse effect on numbers of nuclei attached to the substratum (Fig 7D).

The nature of any polymer surface is determined by interplay between multiple factors mediated by the base monomer and dopant chemistries and modulated by the polymerisation process. With regard to the salient feature of a polymer surface that determine the biomimetic cellular response to contact with the polymer, surface energy, modulus and surface topography interact to yield an overall environment that affects cell/surface interaction [37]. These (amongst other) highly variable influencing factors can make it difficult to identify the precise mechanisms affecting cellular behavior on these polymers. It is noteworthy from this context that the results presented here showed that these relationships can be equally established on dry surfaces immediately post-synthesis as on wetted surfaces representative of the growth environment (Figs 6A and 6B). This provides some indication that these complex effects persist reliably throughout the exposure of the material substrate to the growth environment and good rationale for characterization immediately post-synthesis.

Nevertheless, $i \underline{I t}$ is thus evident from this study that polythiophene chemistry provides a potential mechanism by which the biomimetic properties of biosynthetic scaffolds can be tailored to suit the biological needs of specific cell types (such as the myoblasts used here) if these needs are known. In this study myogenic precursors were observed to react differentially to growth and 
differentiation on various polythiophene surfaces according to monomer and dopant chemistries. With further work aimed towards understanding the precise mechanisms by which polymer and dopant chemistries promote biomimetic effects in specific cell types, this study provides a basis by which myogenic behavior can be controlled by polymer chemistries to attain desired outcomes for skeletal muscle engineering.

\section{Conclusions}

This study has highlighted that polythiophene polymers provide a versatile platform for the directed growth and differentiation of muscle precursors-controlled aceording to monomer and dopant chemistries. Primary myoblasts were observed to proliferate and differentiate on the polymers as effectively as on control (polystyrene and gold-mylar) substrata with the added advantage of being able to modulate myogenic behavior by variation of dopant and incorporating alkoxy modifications to the polymer backbone. The specific mechanism(s) by which these changes in polythiophene chemistry mediate biomimetic responses in myogenic precursors are largely unclear. It is however evident from this study that these changes in myogenic behavior are mediated at least in part, via effects on growth and replication responses of cells growing on the polythiophene surfaces. In turn, these effects appear to be mediated through changes in the physical properties of the polymer, such as roughness and hydrophobicity surface energy, which has more of an effect on cell adhesion than on differentiation, which in turn is more affected by surface roughness than surface energy.

and-Further understanding of ean be exploited by chemical variation to tailor polymer properties to achieve a given desired cellular response.

Further study of the potential of polymer chemistry to effect changes in the molecular biology of the cell should reveal the precise mechanisms by which cell growth and development ean beaffected by the chemical structure and physic-chemical properties of better controlled on biosynthetic scaffolds. These phenomena can potentially be exploited by chemical variation to tailor polymer properties to achieve a given desired cellular response. In turn, this will facilitate the 
developmenthe development of more effective scaffolds by which to replace and/or restore damaged or diseased tissues.

Page 21 


\section{Figure Legends:}

Figure 1. UV-vis spectra of PBTh doped with $\mathrm{ClO}_{4}{ }^{-}$, after electrodeposition after $24 \mathrm{~h}$ in air, after 19 h soaking in culture media 1 and after 24 h soaked in $37^{\circ} \mathrm{C}$ in culture media 2.

Figure 2. AFM imaging of polymer surface topography. $\mathrm{PBTh}_{-} \mathrm{ClO}_{4}$ (A), PBTh-pTS (B), P3DTh$\mathrm{ClO}_{4}(\mathrm{C})$, P3DTh-pTS, PDTTh3E-ClO 4 (E) and PDTTh3E-pTS (F). $\mathrm{ClO}_{4}^{-}$doped polymers were generally rougher than pTS doped polymers reaching statistical significance at $100 \mu \mathrm{m}^{2}$ resolution (G).

Figure 3. Myoblast LDH activity at 24 (A) and 48 (B) h. At 24h (A). At 24 hrs. (A), LDH activity was significantly lower in myoblasts grown on PDTTh3E compared to the remaining two polymers of the $\mathrm{pTS}^{-}$doped group $(p<0.05)$. Myoblasts grown on $\mathrm{ClO}_{4}{ }^{-}$doped polymers did not show any significant difference in LDH activity. By 48 hours (B), differences between polymers were more apparent, indicating variation of effect on cell metabolism according to differences between the pTH formulations. All polymers supported lower LDH activity than gold mylar (Au) surfaces, with significant differences evident between the Au and PBTh-CLO4- and PDTTh3E-pTS surfaces $(p<0.001)$. In the $\mathrm{ClO}_{4}^{-}$doped group, both P3DTh and PDTTh3E had significantly higher activity than PBTh $(p<0.001)$. In the $\mathrm{pTS}^{-}$doped group, P3DTh supported the highest activity, significantly higher than PBTh doped with pTS $\left.^{-}(p<0.01)\right)$. No differences in support of myoblast LDH activity occur between any of the polymer substrata and standard tissue culture polystyrene. In general, the PBTh surfaces (both dopant groups) facilitated less metabolic activity than their similarly doped P3DTh and PDTTh3E counterparts.

Figure 4. Phalloidin labelling of actin filaments demonstrates differences in cell morphology after 24 h growth on the different surfaces. On PBTh polymers (A and B), the majority of cells appear spread out with well-defined actin filaments indicative of pre-fusional myogenic behavior and show similar morphology to cells grown on gold coated mylar $(\mathrm{G})$, while on P3DTh and PDTTh3E cells appeared more rounded with contracted cytoplasm and condensed cytoskeletal arrangement (C-F). Scale bars represent $100 \mu \mathrm{m}$. 
Figure 5. Myoblast differentiation was expressed as the percentage of total nuclei included in multinucleate myofibres containing more than 2 nuclei. Gold coated mylar supported the same level of myoblast differentiation as tissue culture plastic (PS). P3DTh doped with $\mathrm{ClO}_{4}{ }^{-}$and PDTTh3E doped with $\mathrm{pTS}^{-}$and $\mathrm{ClO}_{4}{ }^{-}$showed significantly lower levels of myofibre differentiation than gold mylar and PS ( $p \leq 0.05$, and $p \leq 0.0001$ respectively). Scale bars represent $100 \mu \mathrm{m} .{ }^{*}=\mathrm{p} \leq 0.05$; $* *=\mathrm{p} \leq 0.01 ; * * *=\mathrm{p} \leq 0.001$ and $\# \# \#=\mathrm{p} \leq 0.0001$.

Figure 6. Nuclear density (nuclei per $\mathrm{cm}^{2}$ ) after differentiation followed a similar profile to myoblast differentiation on the various substrata (A). All pTS ${ }^{-}$doped polymers showed significantly higher nuclear density than their $\mathrm{ClO}_{4}^{-}$doped counterparts $(p<0.01$ for PBTh and $p<0.001$ for the others). P3DTh doped with $\mathrm{ClO}_{4}{ }^{-}$and PDTTh3E $\mathrm{ClO}_{4}{ }^{-}$showed significantly lower nuclear density than tissue culture plastic control surfaces (PS) $(p<0.05$ and $p<0.001$ respectively). A significant correlation $(R=0.87)$ was observed between nuclear density and myotube formation $(\mathrm{B})(p<0.03)$. Polymer species were denoted as: PBTH (PB), P3DTh (P3D) and PDTTh3E (PDT3E), with suffixes of $\mathrm{C}$ and pT denoting $\mathrm{ClO}_{4}$ - and pTS dopants respectively.

Figure 7. The effects of surface roughness and hydrophobicity on myoblast behaviour. Trends were observed between contact angle and myoblast adhesion $(p<0.03)(\mathrm{A})$ and myoblast differentiation $(p<0.059$; NS) (B). Correlation trends were also found between RMS and myoblast differentiation (C $p<0.059$; NS) and nuclear density (D, $p<0.02)$.

Supplementary Figure 1. PCNA staining of myoblasts on polymer surfaces (A-F) and gold coated mylar (G). Replicating myoblasts can be seen on all polymer surfaces. Scale bars represent $100 \mu \mathrm{m}$.

\section{Reference List}

[1] Bach AD, Beier JP, Stern-Staeter J, Horch RE. Skeletal muscle tissue engineering. J Cell Mol Med 2004 Oct;8(4):413-22.

[2] Huard J, Roy R, Guerette B, Verreault S, Tremblay G, Tremblay JP. Human myoblast transplantation in immunodeficient and immunosuppressed mice: evidence of rejection. Muscle Nerve 1994 Feb;17(2):224-34.

[3] Gussoni E, Blau HM, Kunkel LM. The fate of individual myoblasts after transplantation into muscles of DMD patients. Nat Med 1997 Sep;3(9):970-7. 
[4] Morandi L, Bernasconi P, Gebbia M, Mora M, Crosti F, Mantegazza R, et al. Lack of mRNA and dystrophin expression in DMD patients three months after myoblast transfer. Neuromuscul Disord 1995 Jul;5(4):291-5.

[5] Huang NF, Thakar RG, Wong M, Kim D, Lee RJ, Li S. Tissue engineering of muscle on micropatterned polymer films. Conf Proc IEEE Eng Med Biol Soc 2004;7:4966-9.

[6] Huang NF, Patel S, Thakar RG, Wu J, Hsiao BS, Chu B, et al. Myotube assembly on nanofibrous and micropatterned polymers. Nano Lett 2006 Mar;6(3):537-42.

[7] Razal JM, Kita M, Quigley AF, Kennedy E, Moulton SE, Kapsa RMI, et al. Wet-Spun Biodegradable Fibers on Conducting Platforms: Novel Architectures for Muscle Regeneration. Adv Funct Mater 2009;(19):3381-8.

[8] Beier JP, Klumpp D, Rudisile M, Dersch R, Wendorff JH, Bleiziffer O, et al. Collagen matrices from sponge to nano: new perspectives for tissue engineering of skeletal muscle. BMC Biotechnol 2009;9:34.

[9] Boldrin L, Elvassore N, Malerba A, Flaibani M, Cimetta E, Piccoli M, et al. Satellite cells delivered by micro-patterned scaffolds: a new strategy for cell transplantation in muscle diseases. Tissue Eng 2007 Feb;13(2):253-62.

[10] Boldrin L, Malerba A, Vitiello L, Cimetta E, Piccoli M, Messina C, et al. Efficient delivery of human single fiber-derived muscle precursor cells via biocompatible scaffold. Cell Transplant 2008;17(5):577-84.

[11] Thorrez L, Shansky J, Wang L, Fast L, VandenDriessche T, Chuah M, et al. Growth, differentiation, transplantation and survival of human skeletal myofibers on biodegradable scaffolds. Biomaterials 2008 Jan;29(1):75-84.

[12] Breukers RD, Gilmore KJ, Kita M, Wagner KK, Higgins MJ, Moulton SE, et al. Creating conductive structures for cell growth: Growth and alignment of myogenic cell types on polythiophenes. J Biomed Mater Res A 2010 Oct;95(1):256-68.

[13] Saxena AK, Marler J, Benvenuto M, Willital GH, Vacanti JP. Skeletal muscle tissue engineering using isolated myoblasts on synthetic biodegradable polymers: preliminary studies. Tissue Eng 1999 Dec;5(6):525-32.

[14] Wang X, Gu X, Yuan C, Chen S, Zhang P, Zhang T, et al. Evaluation of biocompatibility of polypyrrole in vitro and in vivo. J Biomed Mater Res A 2004 Mar 1;68(3):411-22.

[15] Wang Z, Roberge C, Dao LH, Wan Y, Shi G, Rouabhia M, et al. In vivo evaluation of a novel electrically conductive polypyrrole/poly(D,L-lactide) composite and polypyrrolecoated poly(D,L-lactide-co-glycolide) membranes. J Biomed Mater Res A 2004 Jul $1 ; 70(1): 28-38$.

[16] Gilmore KJ, Kita M, Han Y, Gelmi A, Higgins MJ, Moulton SE, et al. Skeletal muscle cell proliferation and differentiation on polypyrrole substrates doped with extracellular matrix components. Biomaterials 2009 Oct;30(29):5292-304.

[17] Bidez PR, III, Li S, MacDiarmid AG, Venancio EC, Wei Y, Lelkes PI. Polyaniline, an electroactive polymer, supports adhesion and proliferation of cardiac myoblasts. J Biomater Sci Polym Ed 2006;17(1-2):199-212. 
[18] Miriani RM, Abidian MR, Kipke DR. Cytotoxic analysis of the conducting polymer PEDOT using myocytes. Conf Proc IEEE Eng Med Biol Soc 2008;2008:1841-4.

[19] Quigley A.F., Razal J.M., Thompson B.C., Moulton S.E., Kita M., Kennedy EL, et al. A Conducting Polymer Platform with Biodegradable Fibers for Stimulation and Guidance of Axonal Growth. Adv Mater 2009;21(43):4393-7.

[20] Richardson RT, Wise AK, Thompson BC, Flynn BO, Atkinson PJ, Fretwell NJ, et al. Polypyrrole-coated electrodes for the delivery of charge and neurotrophins to cochlear neurons. Biomaterials 2009 May;30(13):2614-24.

[21] Richardson RT, Thompson B, Moulton S, Newbold C, Lum MG, Cameron A, et al. The effect of polypyrrole with incorporated neurotrophin-3 on the promotion of neurite outgrowth from auditory neurons. Biomaterials 2007 Jan;28(3):513-23.

[22] Pedrotty DM, Koh J, Davis BH, Taylor DA, Wolf P, Niklason LE. Engineering skeletal myoblasts: roles of three-dimensional culture and electrical stimulation. Am J Physiol Heart Circ Physiol 2005 Apr;288(4):H1620-H1626.

[23] Kawahara Y, Yamaoka K, Iwata M, Fujimura M, Kajiume T, Magaki T, et al. Novel electrical stimulation sets the cultured myoblast contractile function to 'on'. Pathobiology 2006;73(6):288-94.

[24] Langelaan ML, Boonen KJ, Rosaria-Chak KY, van der Schaft DW, Post MJ, Baaijens FP. Advanced maturation by electrical stimulation: Differences in response between C2C12 and primary muscle progenitor cells. J Tissue Eng Regen Med 2011 Jul;5(7):529-39.

[25] Gambhir S, Wagner K, Officer DL. Towards functionalised therthiophene-based polymers. Synthetic Metals 2005 Jul 30;154:117-20.

[26] Neumann T, Hauschka SD, Sanders JE. Tissue engineering of skeletal muscle using polymer fiber arrays. Tissue Eng 2003 Oct;9(5):995-1003.

[27] Jun I, Jeong S, Shin H. The stimulation of myoblast differentiation by electrically conductive sub-micron fibers. Biomaterials 2009 Apr;30(11):2038-47.

[28] Lee PY, Cobain E, Huard J, Huang L. Thermosensitive hydrogel PEG-PLGA-PEG enhances engraftment of muscle-derived stem cells and promotes healing in diabetic wound. Mol Ther 2007 Jun;15(6):1189-94.

[29] Feldhues M, Kampf G, Litterer H, Mecklenburg T, Wegener P. Polyalkoxythiophenes soluble electrically conducting polymers. Synthetic Metals 1989;28(1-2):487-93.

[30] Kapsa RM, Quigley AF, Vadolas J, Steeper K, Ioannou PA, Byrne E, et al. Targeted gene correction in the mdx mouse using short DNA fragments: towards application with bone marrow-derived cells for autologous remodeling of dystrophic muscle. Gene Ther 2002 Jun;9(11):695-9.

[31] Abramoff MD, Magalhaes PJ, Ram SJ. Image Processing with ImageJ. Biophotonics International 2004;11(1):36-42.

[32] Barbarella G, Melucci M, Sotgiu G. The Versatile Thiophene: An Overview of Recent Research on Thiophene-Based Materials. Advanced Materials 2005;17:1581-93. 
[33] Zhou L, Liu X, Xue G. Spectroscopic Studies of the Stability of Doped Polythiophenes Under Air. Spectroscopy Letters 1998;31(7):1529-35.

[34] Daoust G, Leclerc M. Structure-property relationships in alkoxy-substituted polythiophenes. Macromolecules 1991;24(455):459.

[35] Kossmehl G, Niemitz M. Preparation and controlled wetability of poly(2,2'-bithienyl5,5'diyl) layers. Synthetic Metals 2012;41(3):1065-71.

[36] Lampin M, Warocquier C, Legris C, Degrange M, Sigot-Luizard MF. Correlation between substratum roughness and wettability, cell adhesion, and cell migration. J Biomed Mater Res 1997 Jul;36(1):99-108.

[37] Ranella A, Barberoglou M, Bakogianni S, Fotakis C, Stratakis E. Tuning cell adhesion by controlling the roughness and wettability of 3D micro/nano silicon structures. Acta Biomater 2010 Jul;6(7):2711-20.

[38] Fulton AB, Prives J, Farmer SR, Penman S. Developmental reorganization of the skeletal framework and its surface lamina in fusing muscle cells. The Journal of Cell Biology 1981 Oct 1;91(1):103-12.

[39] Griffin MA, Sen S, Sweeney HL, Discher DE. Adhesion-contractile balance in myocyte differentiation. J Cell Sci 2004 Nov 15;117(Pt 24):5855-63.

[40] Nowak SJ, Nahirney PC, Hadjantonakis AK, Baylies MK. Nap1-mediated actin remodeling is essential for mammalian myoblast fusion. J Cell Sci 2009 Sep 15;122(Pt 18):3282-93.

[41] Kim S, Shilagardi K, Zhang S, Hong SN, Sens KL, Bo J, et al. A critical function for the actin cytoskeleton in targeted exocytosis of prefusion vesicles during myoblast fusion. Dev Cell 2007 Apr;12(4):571-86.

[42] Schweikl H, Muller R, Englert C, Hiller KA, Kujat R, Nerlich M, et al. Proliferation of osteoblasts and fibroblasts on model surfaces of varying roughness and surface chemistry. $\mathrm{J}$ Mater Sci Mater Med 2007 Oct;18(10):1895-905.

[43] Balloni S, Calvi EM, Damiani F, Bistoni G, Calvitti M, Locci P, et al. Effects of titanium surface roughness on mesenchymal stem cell commitment and differentiation signaling. Int J Oral Maxillofac Implants 2009 Jul;24(4):627-35.

[44] Lin J, Cai Y, Wang X, Ding B, Yu J, Wang M. Fabrication of biomimetic superhydrophobic surfaces inspired by lotus leaf and silver ragwort leaf. Nanoscale 2011 Mar;3(3):1258-62.

[45] Dowling DP, Miller IS, Ardhaoui M, Gallagher WM. Effect of surface wettability and topography on the adhesion of osteosarcoma cells on plasma-modified polystyrene. J Biomater Appl 2011 Sep;26(3):327-47.

[46] Roncali J. Conjugated poly(thiophenes): Synthesis, functionalization, and applications. Chem Rev 1992;92:711-38.

[47] Higgins MJ, Grosse W, Wagner K, Molino P, Wallace GG. Reversable shape memory of nanoscale deformations in inherently conducting polymers without reprogramming. Physical Chemistry 2011;115(13):3371-8. 